\title{
A method to integrate thermographic data and 3D shapes for Diabetic Foot Disease
}

\author{
by S. Colantonio*, G. Pieri*, O. Salvetti*, M. Benvenuti* ${ }^{\star *}$ S. Barone*** and L. \\ Carassale ${ }^{* * *}$ \\ *Institute of Information Science and Technologies, ISTI-CNR, Pisa, Italy \\ **TDGroup S.p.A., Migliarino Pisano (PI), Italy \\ ***University of Pisa, Dept. of Mechanics, Nuclear and Production Engineering, Pisa, \\ Italy

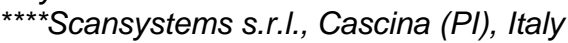

\begin{abstract}
This paper presents the results of a project carried out within a collaboration among TD Group S.p.A., ISTI-CNR, Dept. of Mechanics, Nuclear and Production Engineering, University of Pisa, and the Diabetology Dept. at the "San Giovanni di Dio" Hospital in Florence. The goal of this activity has been to integrate thermal information and three dimensional spatial data by combining acquisitions from a thermocamera and a stereo pair of high resolution visible cameras. An application in the medical field is presented regarding the Diabetic Foot Disease to support physicians in their diagnosis and follow-up.
\end{abstract}

\section{Introduction}

In the frame of a collaboration among TD Group S.p.A., ISTI-CNR, Dept. of Mechanics, Nuclear and Production Engineering, University of Pisa, and the Diabetology Dept. at the "San Giovanni di Dio" Hospital in Florence, an integrated system has been designed and developed for the acquisition and processing of visible and thermal images, and for modeling and reconstructing three-dimensional anatomical structures. The system aims at providing an effective supporting tool for the diagnosis and prognosis of pathologies producing ulceration and laceration, for example that caused by an advanced diabetes condition like the Diabetic Foot Disease (DFD).

The spatial analysis of absolute and relative temperature values can be a valuable support for an early diagnosis and the follow-up monitoring of diabetic patients. It is relevant that, when diabetes is well controlled, it should be possible to avoid serious foot problems, which can otherwise also bring to the lower extremity amputation.

Our integrated system includes the realization of an acquisition device for fusing thermal images [1] with stereo visible high-resolution ones, and for data visualization, registration, and management of three-dimensional reconstructed volumes.

In particular, the 3D volume consists of both the anatomical surface structure, and the related temperature spatial distribution. Both these characteristics support the evaluation of the ulceration, and the identification of anomalies or pathologic conditions.

The paper is organized as follows: in section 2, a description of the aspects related to DFD is given; in section 3 , the materials and methods used to implement 
the system are described; in section 4, a detailed description of the acquisition system is given; in sections 5 and 6 , the implemented algorithms for a single 3D data reconstruction, and the registration of multiple volumes are described. Section 7 describes the patient's data management system, while finally results of experimentations on real case studies are shown in section 8 with some concluding remarks.

\section{Problem specification}

The transfer of IR technologies and related acquisition devices to biomedical imaging allowed to reconsider the diagnostic role and medical applications of the thermal imaging, permitting nowadays to study quantitatively and functionally the thermal property of the human body. Thus, models for the thermal study of human body can be developed to extract quantitative parameters useful in the diagnosis or understanding of thermal control processes in various conditions, for example during the physical activity or as an effect of drug administration.

The use of thermal imaging is usually indicated as functional infrared imaging. Various pathologies can be favorably studied by means of the functional IR imaging, mainly those which present symptoms like an alteration of the normal control of the body temperature, as in particular the cutaneous one. These are dysfunctions of the microcirculation, the macrocirculation in the peripheral districts (figure 1), the peripheral nervous system, local alterations of the tissue metabolism, traumas and phlogosis, already studied in the past by means of the classic medical thermography. Nowadays, these pathologies can be also studied through the evaluation of objective parameters extracted by modeling the temporal evolution and the spatial distribution of the temperature [2].
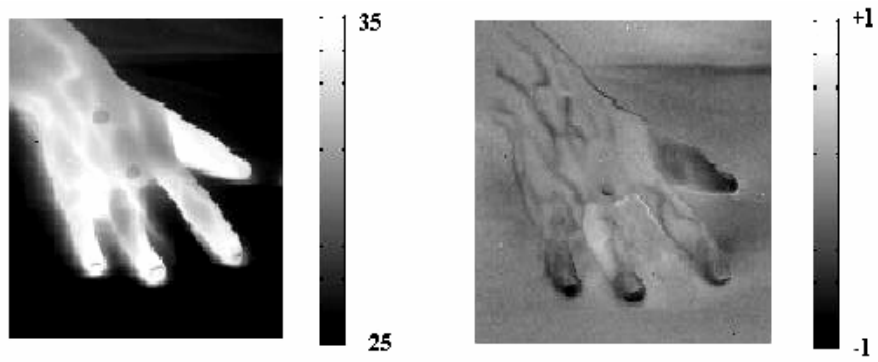

Fig. 1. Functional IR imaging of a patient's hand with circulatory failure. (left), IR image and (right) normalized thermal image to highlight blood vessels

In particular, regarding the DFD pathology, no single broad spectrum antibiotic regimen was shown to be more effective over another in the treatment of diabetic foot ulcers. There is no evidence for the optimal duration or route of antibiotic administration in the cure of diabetic foot ulcers. Treatment of an infected diabetic foot ulcer should be commenced with a broad spectrum antibiotic regimen in conjunction with appropriate debridement. Subsequent antibiotic regimens may be modified with reference to bacteriology and clinical response.

Acute Charcot's foot is associated with a skin temperature $2-8^{\circ} \mathrm{C}$ higher than the contralateral foot as measured on thermography. There is insufficient evidence to recommend the routine use of magnetic resonance imaging or dynamic bone scanning to distinguish acute Charcot's from osteomyelitis. Diagnosis of Charcot's foot should be made by clinical examination supported, where available, by the use 
of thermography. Treatment of Charcot's foot in contact casting is associated with a reduction in skin temperature as measured by thermography and in bone activity as measured by bone isotope uptake compared to the normal foot [3].

In this sense, the use of thermography techniques can be of valuable help for physicians in order to support the diagnosis and especially the follow-up of diabetic patients.

\section{Materials and Methods}

The system is composed of four main parts which are described in deeper details in the following sections:

- Multi-source (visible and infrared) image acquisition

- $\quad 3 D$ reconstruction and integration of stereo visible and thermal data

- $\quad$ Multiple volumes registration

- Data and images management, image visualization and annotation.

The acquisition system is composed of two stereo visible cameras, a synchronized thermocamera, and a structured light projector. From the stereo cameras, a cloud of 3D points is obtained and used for volume reconstruction by building an optimized triangular mesh to model the surface in real colors [4].

Once the object is reconstructed, the thermal information can be associated to each point of the 3D surface. Thus, a 3D model of the investigated structure is obtained, represented with both real and synthesized colors computed from the exact radiance values.

Following single 3D reconstructions, algorithms for multiple volumes registration are used to obtain a complete 3D structure representation, as close as possible to the real object. This is very important to allow physicians to have a global look at the inspected anatomical structure, in such a way that an optimal diagnosis or prognosis can be performed (e.g. all around views of the patient's foot).

Finally, a data management system allows the insertion and management of information related to the specific structure under investigation (e.g. detailed patient identification data), together with the storage of previously reconstructed 3D volumes.

\section{Acquisition system}

The acquisition system is shown in figure 2. The device is composed of a structure equipped with two high resolution visible stereo cameras (SONY XCD 910 SX, 4 Mega pixel resolution), a thermocamera (FLIR A40, wavelength range 7.5 $13 \mu \mathrm{m}$ ), and a structured light projector (NEC LT170) mounted in the centre of the base axis. The mobile support and the horizontal bar mounted on it allow three degrees of freedom. 


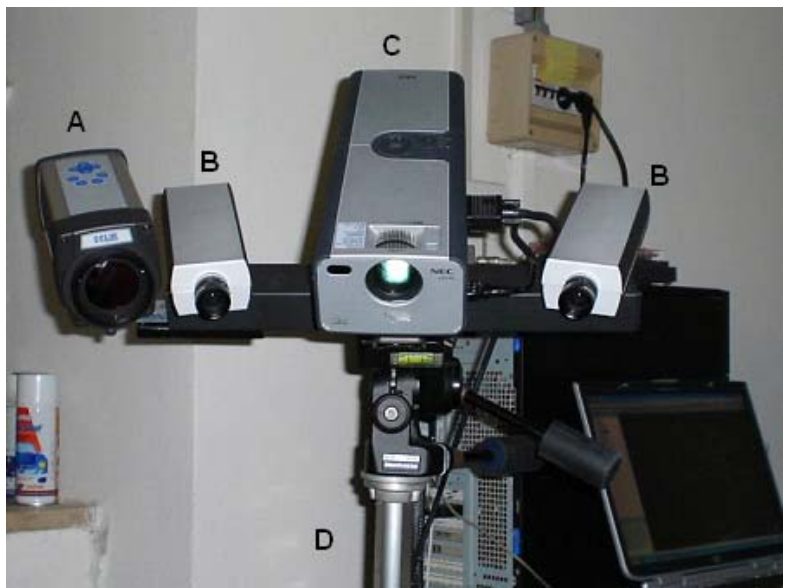

Fig. 2. The acquisition system: $(A)$ thermocamera, $(B)$ visible high resolution stereo cameras, (C) structured light projector, (D) mobile support

The three cameras are connected via a firewire interface (IEEE1394), while the projector is connected through a serial interface (RS232). The projector is used to cast series of regular bands with different width composed of alternate black and white lines, first horizontally and then vertically, in order to capture stereo images at different resolutions and use them to reconstruct the surface of the examined structure. The calibration of the acquisition system is made on a specifically designed sample which can be used with both the visible and IR cameras.

In table 1 the main characteristics of the system are described.

Table 1. Main specifications of the system

\begin{tabular}{|l|l|}
\hline Measurement area $(\mathrm{mm})$ & $280 \times 210$ \\
\hline Acquisition time (sec) & $\leq 20$ \\
\hline N. of measured points & 2000000 \\
\hline Visible cameras resolution & 4 Mega pixel \\
\hline Cameras accuracy $(\mathrm{mm}$ X\&Y axis) & 0.02 \\
\hline Temperature accuracy & 0.03 \\
\hline Thermocamera spectral range $(\mu \mathrm{m})$ & $\pm 2{ }^{\circ} \mathrm{C}$ \\
\hline Thermocamera resolution $($ pixels) & $3.5 \div 13$ \\
\hline Thermal sensitivity $\left({ }^{\circ} \mathrm{C}\right.$ at $\left.30^{\circ} \mathrm{C}\right)$ & $<0.10$ \\
\hline
\end{tabular}

The software implemented for image acquisition, reconstruction, integration, and data management has been developed for Windows platform.

\section{3D Reconstruction and integration}

Stereo reconstruction is based on the same principle as the human visual system uses for depth recovery. With two cameras viewing the same scene under different perspective, geometrical transformations are used for reconstructing 3D structures. Each scene point is projected on different locations in the two cameras and can be localized using the perspective equations [5]. 
The stereoscopic system takes two different views of the observed scene [6].

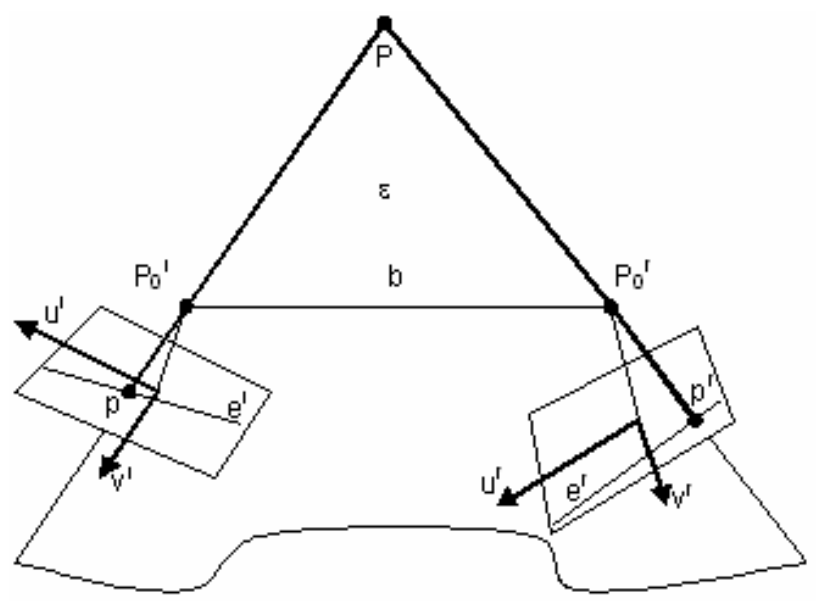

Fig. 3. Geometry of two cameras stereo model

Figure 3 depicts the case of two cameras with identical physical properties. The projection centre of the left image is $P_{0}^{\prime}$, and the projection centre of the right image is $P_{0}^{r}$; the corresponding rotation matrices are $R^{\prime}$ and $R^{r}$. The vector $b=P_{0}^{r}-P_{0}{ }^{\prime}$ $=\left(b_{x}, b_{y}, b_{z}\right)^{T}$ is called stereoscopic base line. If the orientation parameters of both images are known, an object point $P$ can be determined by the intersection of the image rays from two corresponding image points $p^{\prime}$ and $p^{r}$ : each point gives two equations [5] which can be solved for the unknown object co-ordinates $(X, Y, Z)^{T}$. As there are four equations but only three unknowns, the problem is over-determined and can be solved by a least squares adjustment.

An important property of any arrangement of two images of an identical scene and, thus, of all stereo arrangements, is epipolarity: the vectors $b, \overline{P_{0}^{l} P}$ and $\overline{P_{0}^{r} P}$ form a plane called epipolar plane $\varepsilon$. This plane intersects the image planes in the epipolar lines $e^{l}$ and $e^{r}$. The co-planarity condition can be written as follows [7]:

$$
\left(p^{\prime}-p_{0}^{\prime}\right)^{T} \cdot R^{\prime T} \cdot S \cdot R^{r} \cdot\left(p^{r}-p_{0}^{r}\right)=0
$$

with

$$
S=\left(\begin{array}{ccc}
0 & -b_{z} & b_{y} \\
b_{z} & 0 & -b_{x} \\
-b_{y} & b_{x} & 0
\end{array}\right)
$$

and $p_{0}^{\prime}$ and $p_{0}^{r}$ being the projection centres in the camera coordinate systems of the left and right image, respectively. If the orientation parameters of both images are known, for a point $p^{\prime}$ in the left image, the corresponding point $p^{r}$ in the right 
image is situated on the epipolar line $e^{r}$ which is given by Eq. (1). This is an important property which is used in image matching to reduce search space.

Since the thermocamera and the visible cameras are calibrated together the integration phase follows associating thermocamera pixels through specific rotation matrices. In this way the reconstructed structure can be integrated also with the radiance values [8].

\section{Multiple volumes registration}

Once different 3D volumes of the same anatomical structure are reconstructed from different points of view, there are two different ways of registering them into a unique volume representing the whole structure: manually, by selecting specific markers on the different volumes, or automatically, using registration algorithms based on 3D surface shapes. The algorithm for automatic registration is based on the Iterative Closest Point algorithm (ICP) [9]. Correspondences between data sets are computed by matching points in one data set to the closest points in the other data set. A sketch of the algorithm is as follows:

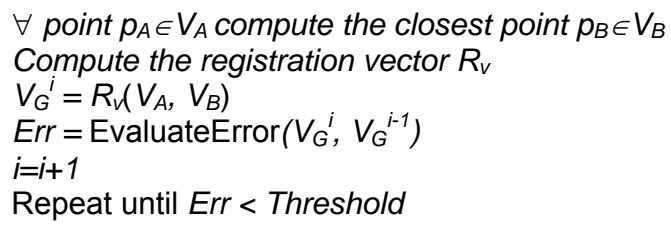

ICP can also handle noise. Since it is an iteration of minimization problem, the algorithm may converge towards a local minimum. Generally it is overcome by starting the iteration loop from an approximate registration. Since we usually wish to compare similar areas captured in similar conditions, the generated 3D volumes are already approximately registered, which is generally sufficient for getting the ICP algorithm to converge without manual intervention. However if these conditions cannot be fulfilled, the registration process is then done in two steps. The first step normalizes the position of the $3 \mathrm{D}$ volumes data using a Procrustes registration. The second one refines the registration using the ICP algorithm [10].

The registration of $3 \mathrm{D}$ volumes allows the projection of their textures in any plane that can be used for their comparison. This means that once the volumes are registered into a unique one, both visible and thermal data can be automatically associated to the structure.

\section{Data management system}

The management of identification data of the patients has been added to the system. Textual data are stored together with the two-dimensional images acquired (both visible and IR), and the 3D reconstruction previously performed. The storage of both visible and IR 3D reconstruction allows a better and deeper medical diagnosis, that can take into account the subjective course of the pathology, and a deep objective statistical analysis linked to the specific therapies for the single pathologies.

\section{Results}




\section{http://dx.doi.org/10.21611/qirt.2006.073}

Several case studies of DFD have been selected by an expert diabetologist and processed to test our system.

In particular, 3D reconstructions have been integrated with radiance values in order to obtain a full description of an examined case. In figure 4 , an example of a single acquired IR image is shown.

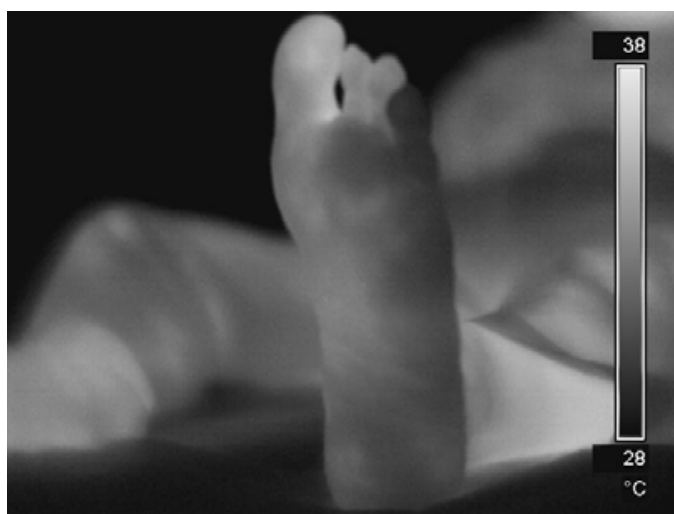

Fig. 4. A single IR image (gray level)

The registration result of multiple volumes of the same foot, related to different shots, is shown in figure 5 .

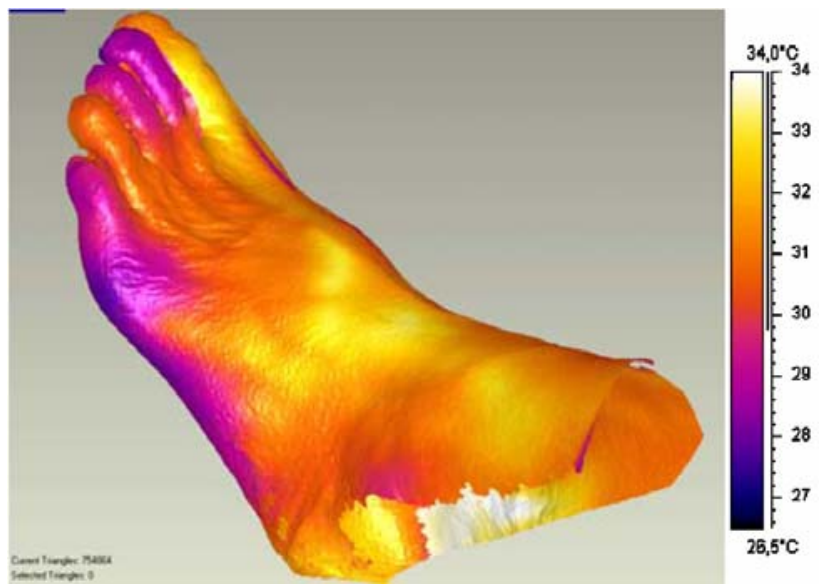

Fig. 5. Example of 3 different acquisitions registered in a single volume and integrated with thermal data (rendered in false colors)

Another example of $3 \mathrm{D}$ reconstruction regarding another case study is shown in figure 6 . The different volumes reconstructed from different acquisitions are registered together to compose a single volume: the feet on the left column, reconstructed from the stereo visible cameras, are visualized in real colors, while the ones on the right are displayed with the radiance (IR) values integrated from the thermocamera. 

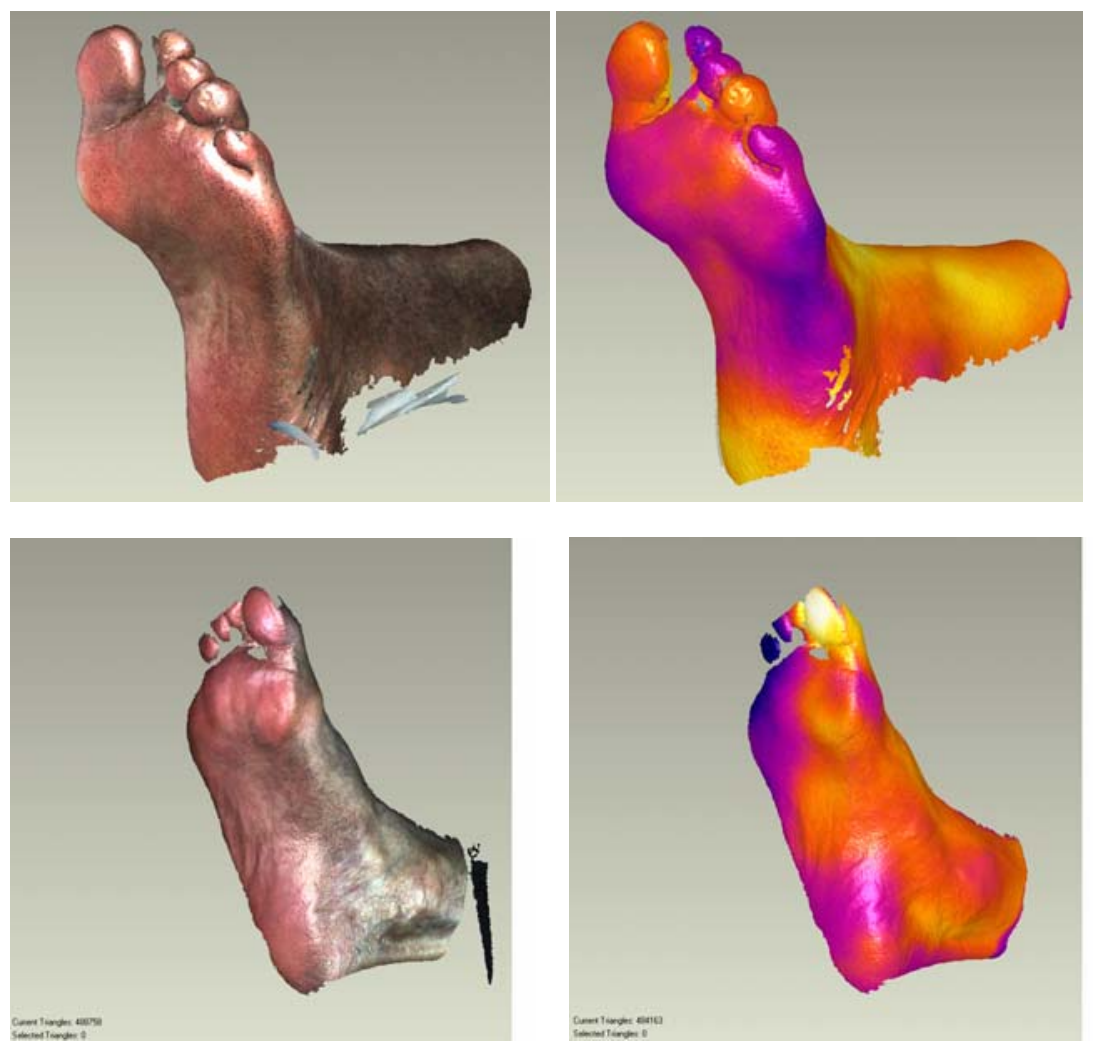

Fig. 6. Two examples of single volumes registered from different acquisitions. A left foot (top), and a right foot (bottom). Real colors from visible cameras (left), and IR false colors (right)

The collaboration with the physicians from the Diabetology Dept. permitted to give more attention to some particular aspects which allowed for an effective improvement of the system in the diagnosis and follow-up of the DFD.

The physicians confirmed that this prototype tool can be a valuable support in their work improving the quality of disease diagnosis and follow-up.

\section{Acknowledgements}

The authors would like to thank Dr. Cristiana Baggiore, Head of the Diabetology Dept. at the "San Giovanni di Dio" Hospital in Florence, for her valuable help and for providing the case studies.

\section{REFERENCES}

[1] S. Colantonio, M.G. Di Bono, G. Pieri, O. Salvetti, M. Benvenuti, "Object tracking in a stereo and infrared vision system", Proceedings of the 8th Advanced Infrared Technologies and Applications Conference - AITA (Rome, Italy, 7-9 September), pp. 113, 2005. 
[2] A. Merla, L. Di Donato, S. Di Luzio, G. Farina, S. Pisarri, M. Proietti, F. Salsano, G.L. Romani, "Infrared Functional Imaging Applied to Raynaud's Phenomenon", IEEE Engineering in Medicine and Biology Magazine, 21 (6), 73-79, 2002.

[3] Raj Mani, A.J. Krentz, C.P. Shearman, "Diabetic Foot Amputation: The Need for an Objective Assessment Tool”, Wounds, 15 (7), 241 - 245, 2003.

[4] C. Rocchini, P. Cignoni, F. Ganovelli, C. Montani, P. Pingi, R. Scopigno, "The Marching Intersections Algorithm for Merging Range Images", The Visual Computer, 20 (2-3), 149 - 164, 2004.

[5] K. Kraus, "Photogrammetry Volume 2. Advanced Methods and Applications", Dümmler Verlag, Bonn, Germany, fourth edition, 1997.

[6] F. Rottensteiner, "Semi-automatic extraction of buildings based on hybrid adjustment using 3D surface models and management of building data in a TIS", PhD Thesis, TU Wien, 2001.

[7] G. Brandstätter, "Notitzen zur voraussetzungslosen gegenseitigen Orientierung von Meßbildern", Österreichische Zeitschrift für Vermessungswesen und Photogrammetrie, 79/4:273-280, 1991.

[8] X. Ju, P. Siebert, "High resolution stereo system", Proc. 8th 3D Modelling Symposium 2003, Paris, France, 2003.

[9] P.J. Besl, N.D. McKay, "A Method of Registration of 3-D Shapes", IEEE Trans. Pattern Analysis and Machine Intelligence, 14 (2), 239-255, 1992.

[10] P. Aksenov, I. Clark, D. Grant, A. Inman, L. Vartikovski and J.-C. Nebel, "3D Thermography for the quantification of heat generation resulting from inflammation", Proc. 8th 3D Modelling Symposium 2003, Paris, France, 2003. 